\title{
Updated Next-to-Next-to-Leading-Order QCD Predictions for the Weak Radiative $B$-Meson Decays
}

\author{
M. Misiak, ${ }^{1}$ H. M. Asatrian, ${ }^{2}$ R. Boughezal,${ }^{3}$ M. Czakon, ${ }^{4}$ T. Ewerth,${ }^{5}$ A. Ferroglia,${ }^{6,7}$ P. Fiedler, ${ }^{4}$ P. Gambino, ${ }^{8}$ \\ C. Greub, ${ }^{9}$ U. Haisch, ${ }^{10,11}$ T. Huber, ${ }^{12}$ M. Kamiński, ${ }^{1}$ G. Ossola, ${ }^{6,7}$ M. Poradziński, ${ }^{1,12}$ A. Rehman, ${ }^{1}$ \\ T. Schutzmeier, ${ }^{13}$ M. Steinhauser, ${ }^{5}$ and J. Virto ${ }^{12}$ \\ ${ }^{1}$ Institute of Theoretical Physics, University of Warsaw, PL-02-093 Warsaw, Poland \\ ${ }^{2}$ Yerevan Physics Institute, 0036 Yerevan, Armenia \\ ${ }^{3}$ High Energy Physics Division, Argonne National Laboratory, Argonne, Illinois 60439, USA \\ ${ }^{4}$ Institut für Theoretische Teilchenphysik und Kosmologie, RWTH Aachen University, D-52056 Aachen, Germany \\ ${ }^{5}$ Institut für Theoretische Teilchenphysik, Karlsruhe Institute of Technology, D-76128 Karlsruhe, Germany \\ ${ }^{6}$ New York City College of Technology, CUNY, Brooklyn, New York 11201, USA \\ ${ }^{7}$ The Graduate School and University Center, CUNY, New York, New York 10016, USA \\ ${ }^{8}$ Dipartimento di Fisica, Università di Torino \& INFN, Torino, I-10125 Torino, Italy \\ ${ }^{9}$ Albert Einstein Center for Fundamental Physics, Institute for Theoretical Physics, University of Bern, CH-3012 Bern, Switzerland \\ ${ }^{10}$ Rudolf Peierls Centre for Theoretical Physics, University of Oxford, OX1 3PN Oxford, United Kingdom \\ ${ }^{11}$ CERN, Theory Division, CH-1211 Geneva 23, Switzerland \\ ${ }^{12}$ Theoretische Physik 1, Naturwissenschaftlich-Technische Fakultät, Universität Siegen, D-57068 Siegen, Germany \\ ${ }^{13}$ Physics Department, Florida State University, Tallahassee, Florida 32306-4350, USA
}

(Received 11 March 2015; published 2 June 2015)

\begin{abstract}
Weak radiative decays of the $B$ mesons belong to the most important flavor changing processes that provide constraints on physics at the $\mathrm{TeV}$ scale. In the derivation of such constraints, accurate standard model predictions for the inclusive branching ratios play a crucial role. In the current Letter we present an update of these predictions, incorporating all our results for the $\mathcal{O}\left(\alpha_{s}^{2}\right)$ and lower-order perturbative corrections that have been calculated after 2006. New estimates of nonperturbative effects are taken into account, too. For the $C P$ - and isospin-averaged branching ratios, we find $\mathcal{B}_{s \gamma}=(3.36 \pm 0.23) \times 10^{-4}$ and $\mathcal{B}_{d \gamma}=\left(1.73_{-0.22}^{+0.12}\right) \times 10^{-5}$, for $E_{\gamma}>1.6 \mathrm{GeV}$. Both results remain in agreement with the current experimental averages. Normalizing their sum to the inclusive semileptonic branching ratio, we obtain $R_{\gamma} \equiv\left(\mathcal{B}_{s \gamma}+\mathcal{B}_{d \gamma}\right) / \mathcal{B}_{c \ell \nu}=(3.31 \pm 0.22) \times 10^{-3}$. A new bound from $\mathcal{B}_{s \gamma}$ on the charged Higgs boson mass in the two-Higgs-doublet-model II reads $M_{H^{ \pm}}>480 \mathrm{GeV}$ at $95 \%$ C.L.
\end{abstract}

Introduction.-The inclusive decays $\bar{B} \rightarrow X_{s} \gamma$ and $\bar{B} \rightarrow$ $X_{d} \gamma$ are considered among the most interesting flavor changing neutral current processes. They contribute in a significant manner to current bounds on masses and interactions of possible additional Higgs bosons and/or supersymmetric particles. The evaluation of such bounds depends in a crucial manner on both the central values and uncertainties of the branching ratio predictions within the standard model (SM). Updating the SM predictions is the main purpose of the present Letter.

Measurements of the $C P$ - and isospin-averaged $\bar{B} \rightarrow$ $X_{s} \gamma$ branching ratio by CLEO [1], Belle [2,5], and BABAR [6-9] lead to the combined result [4]

$$
\mathcal{B}_{s \gamma}^{\exp }=(3.43 \pm 0.21 \pm 0.07) \times 10^{-4},
$$

for the photon energy $E_{\gamma}>E_{0}=1.6 \mathrm{GeV}$ in the decaying meson rest frame. The combination involves an extrapolation from measurements performed at $E_{0} \in[1.7,2.0] \mathrm{GeV}$. Applying the same extrapolation method to the available $\bar{B} \rightarrow X_{d} \gamma$ measurement [10], one finds

$$
\mathcal{B}_{d \gamma}^{\exp }=(1.41 \pm 0.57) \times 10^{-5}
$$

at $E_{0}=1.6 \mathrm{GeV}$ [11]. More precise determinations of $\mathcal{B}_{q \gamma}^{\exp }$ for $q=s, d$ are expected from Belle II [12].

Theoretical calculations of $\mathcal{B}_{q \gamma}$ have a chance to match the experimental precision only in a certain range of $E_{0}$ where the nonperturbative contribution $\delta \Gamma_{\text {nonp }}$ in the relation

$$
\Gamma\left(\bar{B} \rightarrow X_{q} \gamma\right)=\Gamma\left(b \rightarrow X_{q}^{p} \gamma\right)+\delta \Gamma_{\text {nonp }}
$$

remains under control. Here, $\Gamma\left(b \rightarrow X_{q}^{p} \gamma\right)$ denotes the perturbatively calculable rate of the radiative $b$-quark decay involving only charmless partons in the final state. Their overall strangeness vanishes for $X_{d}^{p}$ and equals -1 for $X_{s}^{p}$. The analysis of Ref. [13] implies that unknown contributions to $\delta \Gamma_{\text {nonp }}$ are potentially larger than the so-far determined ones, and induce around $\pm 5 \%$ uncertainty in $\mathcal{B}_{s \gamma}$ at $E_{0}=1.6 \mathrm{GeV}$. Nonperturbative uncertainties in $\mathcal{B}_{d \gamma}$ receive additional sizeable contributions [14] due to collinear photon emission in the $b \rightarrow d u \bar{u} \gamma$ process whose 
Cabibbo-Kobayashi-Maskawa (CKM) factor is only a few times smaller than the one in the leading term.

Apart from possible future progress in analyzing nonperturbative effects, one needs to determine $\Gamma\left(b \rightarrow X_{q}^{p} \gamma\right)$ to a few percent accuracy. It requires evaluating next-to-nextto-leading order (NNLO) QCD corrections that involve Feynman diagrams up to four loops. The first SM estimate of the $\bar{B} \rightarrow X_{s} \gamma$ branching ratio at this level was presented in Ref. [15] where all the corrections calculated up to 2006 were taken into account. A part of the $\mathcal{O}\left(\alpha_{s}^{2}\right)$ contribution was obtained via interpolation [16] in the charm quark mass between the large- $m_{c}$ asymptotic expression [17] and the $m_{c}=0$ boundary condition that was estimated using the Brodsky-Lepage-Mackenzie (BLM) approximation [18].

In the present Letter, we provide an updated prediction for $\mathcal{B}_{s \gamma}$, including all the contributions and estimates worked out after the completion of Ref. [15]. They are listed in the next section where the necessary definitions are introduced. The interpolation in $m_{c}$ is still being applied. However, the $m_{c}=0$ boundary condition is no longer a BLM-based estimate but rather comes from an explicit calculation [19].

The current analysis supersedes our previous one in Ref. [15], which was published in 2006 and has not been updated since then. It has been widely considered as a standard reference until now. The time for our update comes only at present because the most recent and technically challenging four-loop calculation of Ref. [19] constitutes a breakthrough in the analysis. It has an important effect on the central value of $\mathcal{B}_{s \gamma}$.

The Letter is organized as follows: After discussing $\mathcal{B}_{s \gamma}$, our NNLO analysis is extended to $\mathcal{B}_{d \gamma}$. Next, we consider $R_{\gamma} \equiv\left(\mathcal{B}_{s \gamma}+\mathcal{B}_{d \gamma}\right) / \mathcal{B}_{c \ell \nu}$ which may sometimes be more convenient than $\mathcal{B}_{s \gamma}$ for deriving constraints on new physics. Finally, we present a generic expression for beyond-SM contributions, as well as an updated bound for the charged Higgs boson mass in the two-Higgsdoublet-model II (THDM II).

$\mathcal{B}_{s \gamma}$ in the SM.-Radiative $B$-meson decays are most conveniently described in the framework of an effective theory that arises after decoupling of the $W$ boson and heavier particles. Flavor-changing weak interactions that are relevant for $\Gamma\left(b \rightarrow X_{q}^{p} \gamma\right)$ with $q=s, d$ are given by

$$
\mathcal{L}_{\text {eff }} \sim V_{t q}^{*} V_{t b}\left[\sum_{i=1}^{8} C_{i} Q_{i}+\kappa_{q} \sum_{i=1}^{2} C_{i}\left(Q_{i}-Q_{i}^{u}\right)\right] .
$$

Explicit expressions for the current-current $\left(Q_{1,2}\right)$, fourquark penguin $\left(Q_{3, \ldots, 6}\right)$, photonic dipole $\left(Q_{7}\right)$, and gluonic dipole $\left(Q_{8}\right)$ operators can be found, e.g., in Eq. (2.5) of Ref. [16]. The CKM element ratio $\kappa_{q}=\left(V_{u q}^{*} V_{u b}\right) /$ $\left(V_{t q}^{*} V_{t b}\right)$ is small for $q=s$, and it affects $\mathcal{B}_{s \gamma}$ by less than $0.3 \%$. Barring this effect and the higher-order electroweak ones, $\Gamma\left(b \rightarrow X_{s}^{p} \gamma\right)$ in the $\mathrm{SM}$ is given by a quadratic polynomial in the real Wilson coefficients $C_{i}$

$$
\Gamma\left(b \rightarrow X_{s}^{p} \gamma\right) \sim \sum_{i, j=1}^{8} C_{i} C_{j} G_{i j}
$$

A series of contributions to the above expression from our calculations in Refs. [19-28] makes the current analysis significantly improved with respect to the one in Ref. [15]. In particular, the NNLO Wilson coefficient calculation becomes complete after including the four-loop anomalous dimensions that describe $Q_{1, \ldots, 6} \rightarrow Q_{8}$ mixing under renormalization [20]. Effects of the charm and bottom quark masses in loops on the gluon lines in $G_{77}$ [21], $G_{78}$ [22], and $G_{(1,2) 7}$ [23], as well as a complete calculation of $G_{78}$ [24], are now available. Three- and four-body finalstate contributions to $G_{88}[25,26]$ and $G_{(1,2) 8}$ [26] are included in the BLM approximation. Four-body final-state contributions involving the penguin and $Q_{1,2}^{u}$ operators are taken into account at the leading order (LO) [27] and next-to-leading order (NLO) [28]. Last but not least, the complete NNLO calculation [19] of $G_{17}$ and $G_{27}$ at $m_{c}=0$ is used as a boundary for interpolating their unknown parts in $m_{c}$.

Following the algorithm described in detail in Ref. [19], taking into account new nonperturbative effects [13,29,30], as well as the previously omitted parts of the NNLO BLM corrections [31], we arrive at the following SM prediction

$$
\mathcal{B}_{s \gamma}^{\mathrm{SM}}=(3.36 \pm 0.23) \times 10^{-4} \text { for } E_{0}=1.6 \mathrm{GeV} .
$$

Individual contributions to the total uncertainty are of nonperturbative $( \pm 5 \%)$, higher-order $( \pm 3 \%)$, interpolation $( \pm 3 \%)$, and parametric $( \pm 2 \%)$ origin. They are combined in quadrature. The parametric one gets reduced with respect to Ref. [15], which becomes possible thanks to the new semileptonic fits of Ref. [32]. Our input parameters, their uncertainties, and the corresponding correlation matrix can be found in Appendix D of Ref. [19]. Since we normalize to the semileptonic branching ratio $\mathcal{B}_{c \ell \nu}$, our result shows little sensitivity to the $b$-quark mass and the CKM angles. The main parametric uncertainty $( \pm 1.5 \%)$ originates from $\mathcal{B}_{c \ell \nu}$, while the next one $( \pm 0.75 \%)$ comes from $\alpha_{s}\left(M_{Z}\right)$.

As far as the interpolation uncertainty is concerned, one might have hoped for its reduction with respect to Ref. [15] after the explicit evaluation of the $m_{c}=0$ boundary [19]. Unfortunately, the interpolated parts of the $\mathcal{O}\left(\alpha_{s}^{2}\right)$ contributions to $G_{(1,2) 7}$ turn out to be sizeable. Their effect on $\mathcal{B}_{s \gamma}^{\mathrm{SM}}$ grows from $0 \%$ to around $5 \%$ when $m_{c}$ changes from 0 up to the measured value (see Fig. 4 of Ref. [19]). In such a situation, we prefer to stay conservative, and retain our interpolation uncertainty estimate at the $\pm 3 \%$ level.

For the higher-order uncertainty estimation, it is useful to study how $\mathcal{B}_{s \gamma}^{\mathrm{SM}}$ depends on three renormalization scales: the matching scale $\mu_{0} \sim m_{t}$ at which the heavy particles $(t$, $W, Z, H^{0}$ ) are decoupled, the low-energy scale $\mu_{b} \sim m_{b} / 2$ at which the Wilson coefficient renormalization group evolution is terminated, and the scale $\mu_{c}$ at which the 
charm quark mass is renormalized. We vary them in the ranges $\mu_{0} \in[80,320] \mathrm{GeV}$ and $\mu_{b}, \mu_{c} \in[1.25,5] \mathrm{GeV}$, setting the central values to $\mu_{0}=160 \mathrm{GeV}$ and $\mu_{b}=\mu_{c}=2 \mathrm{GeV}$. The observed scale dependence (see Fig. 6 of Ref. [19]) turns out to be quite similar to the one in Fig. 2 of Ref. [15]. Therefore, we leave the higher-order uncertainty estimate at the $\pm 3 \%$ level, i.e., unchanged with respect to Ref. [15].

The nonperturbative uncertainty estimate of $\pm 5 \%$ is adopted from Ref. [13] without any modification. It turns out to be identical to our earlier rough estimate in Ref. [15]. Some comments on possible future suppression of this uncertainty are given in our summary below.

The central value in Eq. (6) is considerably higher than $3.15 \times 10^{-4}$ in Ref. [15], although the difference between the two values does not exceed the previously estimated uncertainty. A detailed description of various contributions to this difference is given in Sec. 4 of Ref. [19], as well as in Table 2 there.

$\mathcal{B}_{d \gamma}$ in the SM.-Extending our NNLO calculation to the $\mathcal{B}_{d \gamma}$ case begins with inserting the proper CKM factors in Eq. (4). Contrary to $\kappa_{s}$, the ratio $\kappa_{d}$ is not numerically small. Using the CKM fits of Ref. [33], one finds

$$
\kappa_{d}=\left(0.007_{-0.011}^{+0.015}\right)+i\left(-0.404_{-0.014}^{+0.012}\right) .
$$

The small real part implies that the effects of $\kappa_{d}$ on the $C P$ averaged $\mathcal{B}_{d \gamma}$ are dominated by those proportional to $\left|\kappa_{d}\right|^{2}$. In such terms, perturbative two- and three-body final state contributions arise only at the NNLO and NLO, respectively. They vanish in the $m_{c}=m_{u}$ limit, which effectively makes them suppressed by $m_{c}^{2} / m_{b}^{2} \lesssim 0.1$. In consequence, the main $\kappa_{d}$ effect comes from $b \rightarrow d u \bar{u} \gamma$ at the LO, where phase-space suppression is partially compensated by the collinear logarithms.

In the first (rough) approximation, one evaluates the treelevel $b \rightarrow d u \bar{u} \gamma$ diagrams retaining a common light-quark mass $m_{q}$ inside the collinear logarithms [26], and varying $m_{b} / m_{q}$ between $10 \sim m_{B} / m_{K}$ and $50 \sim m_{B} / m_{\pi}$ to estimate the uncertainty. The considered effect varies then from $2 \%$ to $11 \%$ of $\mathcal{B}_{d \gamma}$. A more involved analysis with the help of fragmentation functions gives a very similar range [14]. Including this contribution in our evaluation of the entire $B_{d \gamma}$ from Eq. (4), we find

$$
\mathcal{B}_{d \gamma}^{\mathrm{SM}}=\left(1.73_{-0.22}^{+0.12}\right) \times 10^{-5} \text { for } E_{0}=1.6 \mathrm{GeV},
$$

where the central value corresponds to $m_{b} / m_{q}=50$. Our result is about $12 \%$ larger than the one given in Ref. [11] where the $b \rightarrow d u \bar{u} \gamma$ contributions were neglected. The uncertainty estimate in Eq. (8) improves with respect to Ref. [11] thanks to including the NNLO QCD corrections and using the updated CKM fit [33]. Interestingly, the parametric uncertainty due to the CKM input amounts to $\pm 2.5 \%$ only.
The collinear logarithm problem might seem artificial because isolated photons are required in the experimental signal sample. Unfortunately, requiring photon isolation on the perturbative side would necessitate introducing an infrared cutoff on the gluon energies, e.g., in the NLO corrections to the dominant $G_{77}$ term. Without a dedicated analysis (which is beyond the scope of the present Letter), it is hard to verify whether such an approach would enhance or suppress the uncertainty in $\mathcal{B}_{d \gamma}$.

Another question concerning the $\left|\kappa_{d}\right|^{2}$ terms is whether the off-shell light vector meson conversion to photons can be assumed to be included in our overall $\pm 5 \%$ nonperturbative uncertainty. Much smaller effects found in the vector-meson-dominance analysis of Ref. [34] imply that it is likely to be the case.

The ratio $R_{\gamma}$. - In the fully inclusive measurements of radiative $B$-meson decays [1,5-8], the final hadronic state strangeness is not verified. The actually measured quantity is $\mathcal{B}_{s \gamma}+\mathcal{B}_{d \gamma}$. Next, the result is divided by $\left(1+\left|\left(V_{t d}^{*} V_{t b}\right)\right|\right.$ $\left.\left.\left(V_{t s}^{*} V_{t b}\right)\right|^{2}\right)$ to obtain $\mathcal{B}_{s \gamma}$. To avoid such a complication, we provide here our SM prediction for $\mathcal{B}_{s \gamma}+\mathcal{B}_{d \gamma}$ with all the correlated uncertainties properly taken into account. Moreover, we normalize it to the $C P$ - and isospin-averaged inclusive semileptonic branching ratio $\mathcal{B}_{c \ell \nu}$. In the $\mathcal{B}_{s \gamma}$ case, such a normalization reduces the parametric uncertainty from $\pm 2.0 \%$ to $\{+1.2,-1.4\} \%$. It may also be useful on the experimental side because the inclusive semileptonic events can serve for determining the $B$-meson yield. Proceeding as in the previous sections, we obtain for $E_{\gamma}=1.6 \mathrm{GeV}$

$$
R_{\gamma}^{\mathrm{SM}} \equiv\left(\mathcal{B}_{s \gamma}^{\mathrm{SM}}+\mathcal{B}_{d \gamma}^{\mathrm{SM}}\right) / \mathcal{B}_{c \ell \nu}=(3.31 \pm 0.22) \times 10^{-3} .
$$

The relative uncertainties are identical to those in $\mathcal{B}_{s \gamma}$ [as given below Eq. (6)], except for the parametric one, which amounts to $\{+1.2,-1.7\} \%$ including the effect of $m_{b} / m_{q}$. The gain in the overall theory uncertainty is hardly noticeable, but this may change with the future progress in determining the perturbative and nonperturbative corrections.

Beyond-SM effects.-In most of the new-physics scenarios considered in the literature, beyond-SM effects on $\mathcal{B}_{s \gamma}$ are driven by new additive contributions to the Wilson coefficients of the dipole operators at the matching scale $\mu_{0}$. Denoting such contributions by $\Delta C_{7,8}$ and setting $\mu_{0}$ to $160 \mathrm{GeV}$, we find

$$
\begin{aligned}
\mathcal{B}_{s \gamma} \times 10^{4} & =(3.36 \pm 0.23)-8.22 \Delta C_{7}-1.99 \Delta C_{8}, \\
R_{\gamma} \times 10^{3} & =(3.31 \pm 0.22)-8.05 \Delta C_{7}-1.94 \Delta C_{8} .
\end{aligned}
$$

The above expressions are linearized; i.e., it is assumed that the quadratic terms in $\Delta C_{7,8}$ are negligible when they enter with $\mathcal{O}(1)$ coefficients into the above equations. If they are not, a detailed analysis of QCD corrections in the considered beyond-SM scenario is necessary. 
Such an analysis is available in the THDM II [35] for which the NLO [36-38] and NNLO [39] corrections to $\Delta C_{7,8}$ are known. They are always negative and remain practically independent of the vacuum expectation value ratio $\tan \beta$ when $\tan \beta \gtrsim 2$. Sending $\tan \beta$ to infinity in the expressions for $\Delta C_{7,8}$, we find the following updated bounds from $\mathcal{B}_{s \gamma}$ on the charged Higgs boson mass in this model

$$
\begin{array}{ll}
M_{H^{ \pm}}>480 \mathrm{GeV} & \text { at } 95 \% \text { C.L. }, \\
M_{H^{ \pm}}>358 \mathrm{GeV} & \text { at } 99 \% \text { C.L. }
\end{array}
$$

For $\tan \beta \lesssim 2$ the bounds become considerably stronger, but at the same time other observables provide competitive limits [40]. In the supersymmetric case, in which the charged scalar and the neutral pseudoscalar tend to be almost degenerate, the current direct search bounds [41,42] exceed $500 \mathrm{GeV}$ for $\tan \beta \gtrsim 20$.

Summary.-We presented an updated prediction for $\mathcal{B}_{s \gamma}$ in the SM taking into account all the perturbative and nonperturbative effects worked out after the 2006 publication [15] of the first NNLO estimate for this quantity. Our current analysis supersedes the one of Ref. [15].

Some of the $\mathcal{O}\left(\alpha_{s}^{2}\right)$ corrections are still interpolated in $m_{c}$, but the $m_{c}=0$ boundary condition now comes from an explicit calculation. Despite this improvement, the interpolation uncertainty cannot be reduced because the interpolated correction is sizeable. Future progress requires extending the calculation of $G_{(1,2) 7}$ to arbitrary $m_{c}$, which is considered a difficult but manageable task. It would amount to evaluating the same propagator diagrams with unitarity cuts as in Ref. [19], but for arbitrary $m_{c}$ rather than just for $m_{c}=0$. Several hundreds of four-loop two-scale master integrals would need to be calculated. For this purpose, one could numerically solve differential equations in the variable $z=m_{c}^{2} / m_{b}^{2}$. The necessary boundary conditions at $z \gg 1$ could be found from asymptotic expansions in this limit. Determining such boundary conditions involves only three-loop single-scale propagator integrals. They are likely much simpler than the four-loop single-scale ones in Ref. [19].

In parallel, one should investigate whether nonperturbative uncertainties can be suppressed by combining lattice inputs with measurements of observables like the $C P$ or isospin asymmetries in $\bar{B} \rightarrow X_{q} \gamma$. In the analysis of Ref. [13], nonperturbative effects have been parametrized in terms of the so-called subleading shape functions, i.e., matrix elements of nonlocal operators between the $B$-meson states at rest. Determining such functions directly seems to remain beyond the current lattice capabilities. However, constraints on them can be derived from matrix elements of local operators, the same ones that matter for the extraction of $\left|V_{c b}\right|$ from $\mathcal{B}_{c \ell \nu}$ [32]. The higherdimensional operator matrix elements are practically unconstrained by the data. Any lattice estimates of them could help to suppress the nonperturbative uncertainties in both $\left|V_{c b}\right|$ and $\mathcal{B}_{s \gamma}$.

The main outcome of our current analysis is an upwards shift by around $6.4 \%$ in the central value of $\mathcal{B}_{s \gamma}^{\mathrm{SM}}$. It originates mainly from fixing the $m_{c}=0$ boundary $(+3 \%)$ and including the complete NNLO BLM corrections to the three- and four-body final state channels $(+2 \%)$. Both effects are within the previously [15] estimated interpolation $( \pm 3 \%)$ and higher-order $( \pm 3 \%)$ uncertainties, respectively. Nevertheless, the obtained $\mathcal{O}(1 \sigma)$ increase of the central value is an important one, especially in the context of constraining beyond-SM theories. The new four-loop calculation of the $m_{c}=0$ boundary in Ref. [19] improves an essential point in the analysis, and brings the estimated NNLO effects under much better control.

Since $\mathcal{B}_{s \gamma}^{\text {SM }}$ is now closer to $\mathcal{B}_{s \gamma}^{\text {exp }}$ (but still $\mathcal{B}_{s \gamma}^{\text {SM }}<\mathcal{B}_{s \gamma}^{\text {exp }}$ ), the bound on $M_{H^{ \pm}}$in the THDM II becomes significantly stronger. The $95 \%$ C.L. one grows by $120 \mathrm{GeV}$ with respect to its previous evaluation in Ref. [39] (cf. "note added" there). For moderate values of $\tan \beta$, no other available measurement constrains $M_{H^{ \pm}}$in a more efficient manner.

We supplemented our analysis with new NNLO predictions for $\mathcal{B}_{d \gamma}$ and for the ratio $R_{\gamma}=\left(\mathcal{B}_{s \gamma}+\mathcal{B}_{d \gamma}\right) / \mathcal{B}_{c \ell v}$ where correlated uncertainties are treated in a consistent manner. The ratio $R_{\gamma}$ may serve in the future as a more convenient observable for testing beyond-SM theories with minimal flavor violation.

We acknowledge partial support from the Deutsche Forschungsgemeinschaft (DFG) within the research unit FOR 1873 (QFET) and within the Sonderforschungsbereich Transregio 9 "Computational Particle Physics," from the State Committee of Science of Armenia Program No 13-1c153 and Volkswagen Stiftung Program No 86426, from the Swiss National Science Foundation, from the National Science Centre (Poland) research project, Decision No. DEC-2014/13/B/ST2/03969, from the U.S. Department of Energy, Division of High Energy Physics, under Contract No. DE-AC02-06CH11357, from the U.S. National Science Foundation under Grant No. PHY-1417354, and from MIUR under Contract No. 2010YJ2NYW 006.

[1] S. Chen et al. (CLEO Collaboration), Phys. Rev. Lett. 87, 251807 (2001).

[2] K. Abe et al. (Belle Collaboration), Phys. Lett. B 511, 151 (2001). This measurement has recently been superseded by a new one in Ref. [3], which is not yet taken into account in the world average of Ref. [4].

[3] T. Saito et al. (Belle Collaboration), Phys. Rev. D 91, 052004 (2015).

[4] Y. Amhis et al. (Heavy Flavor Averaging Group), arXiv:1412.7515. 
[5] A. Limosani et al. (Belle Collaboration), Phys. Rev. Lett. 103, 241801 (2009).

[6] B. Aubert et al. (BABAR Collaboration), Phys. Rev. D 77, 051103 (2008).

[7] J. P. Lees et al. (BABAR Collaboration), Phys. Rev. Lett. 109, 191801 (2012).

[8] J. P. Lees et al. (BABAR Collaboration), Phys. Rev. D 86, 112008 (2012).

[9] J. P. Lees et al. (BABAR Collaboration), Phys. Rev. D 86, 052012 (2012).

[10] P. del Amo Sanchez et al. (BABAR Collaboration), Phys. Rev. D 82, 051101 (2010).

[11] A. Crivellin and L. Mercolli, Phys. Rev. D 84, 114005 (2011).

[12] T. Aushev et al., arXiv:1002.5012.

[13] M. Benzke, S. J. Lee, M. Neubert, and G. Paz, J. High Energy Phys. 08 (2010) 099.

[14] H. M. Asatrian and C. Greub, Phys. Rev. D 88, 074014 (2013).

[15] M. Misiak et al., Phys. Rev. Lett. 98, 022002 (2007).

[16] M. Misiak and M. Steinhauser, Nucl. Phys. B764, 62 (2007).

[17] M. Misiak and M. Steinhauser, Nucl. Phys. B840, 271 (2010).

[18] S. J. Brodsky, G. P. Lepage, and P. B. Mackenzie, Phys. Rev. D 28, 228 (1983).

[19] M. Czakon, P. Fiedler, T. Huber, M. Misiak, T. Schutzmeier, and M. Steinhauser, J. High Energy Phys. 04 (2015) 168.

[20] M. Czakon, U. Haisch, and M. Misiak, J. High Energy Phys. 03 (2007) 008.

[21] H. M. Asatrian, T. Ewerth, H. Gabrielyan, and C. Greub, Phys. Lett. B 647, 173 (2007).

[22] T. Ewerth, Phys. Lett. B 669, 167 (2008).

[23] R. Boughezal, M. Czakon, and T. Schutzmeier, J. High Energy Phys. 09 (2007) 072.
[24] H. M. Asatrian, T. Ewerth, A. Ferroglia, C. Greub, and G. Ossola, Phys. Rev. D 82, 074006 (2010).

[25] A. Ferroglia and U. Haisch, Phys. Rev. D 82, 094012 (2010).

[26] M. Misiak and M. Poradziński, Phys. Rev. D 83, 014024 (2011).

[27] M. Kamiński, M. Misiak, and M. Poradziński, Phys. Rev. D 86, 094004 (2012).

[28] T. Huber, M. Poradziński, and J. Virto, J. High Energy Phys. 01 (2015) 115.

[29] T. Ewerth, P. Gambino, and S. Nandi, Nucl. Phys. B830, 278 (2010).

[30] A. Alberti, P. Gambino, and S. Nandi, J. High Energy Phys. 01 (2014) 147.

[31] Z. Ligeti, M. E. Luke, A. V. Manohar, and M. B. Wise, Phys. Rev. D 60, 034019 (1999).

[32] A. Alberti, P. Gambino, K. J. Healey, and S. Nandi, Phys. Rev. Lett. 114, 061802 (2015).

[33] J. Charles et al. (CKMfitter Group Collaboration), Phys. Rev. D 91, 073007 (2015).

[34] G. Ricciardi, Phys. Lett. B 355, 313 (1995).

[35] L. F. Abbott, P. Sikivie, and M. B. Wise, Phys. Rev. D 21, 1393 (1980).

[36] M. Ciuchini, G. Degrassi, P. Gambino, and G. F. Giudice, Nucl. Phys. B527, 21 (1998).

[37] F. M. Borzumati and C. Greub, Phys. Rev. D 58, 074004(1998).

[38] F. M. Borzumati and C. Greub, Phys. Rev. D 59, 057501 (1999).

[39] T. Hermann, M. Misiak, and M. Steinhauser, J. High Energy Phys. 11 (2012) 036.

[40] O. Eberhardt, U. Nierste, and M. Wiebusch, J. High Energy Phys. 07 (2013) 118.

[41] V. Khachatryan et al. (CMS Collaboration), J. High Energy Phys. 10 (2014) 160.

[42] G. Aad et al. (ATLAS Collaboration), J. High Energy Phys. 11 (2014) 056 